\title{
DOC-2/DAB2 interactive protein regulates proliferation and mobility of nasopharyngeal carcinoma cells by targeting PI3K/Akt pathway
}

\author{
BIN WANG ${ }^{1}$, QIANQIAN GU ${ }^{2}$ and $\mathrm{JIAN} \mathrm{LI}^{1}$ \\ Departments of ${ }^{1}$ Otorhinolaryngology and ${ }^{2}$ Geriatrics, Cangzhou Central Hospital, Cangzhou, Hebei 061000, P.R. China
}

Received December 20, 2016; Accepted May 24, 2017

DOI: $10.3892 /$ or.2017.5704

\begin{abstract}
Tumor suppressors are a group of important inverse regulators for carcinogenesis in human cancers including nasopharyngeal carcinoma (NPC). DOC-2/DAB2 interactive protein (DAB2IP) has been found to be a novel tumor suppressor in malignancies. However, the expression and biological function of DAB2IP in NPC have not been previously reported. This study found that the levels of DAB2IP were decreased in NPC tissues compared to non-cancerous nasopharyngeal epithelium tissues. In addition, downregulation of DAB2IP mRNA was confirmed by qRT-PCR in NPC cell lines as compared with a human immortalized nasopharyngeal epithelial cell line NP69. The reduced expression of DAB2IP was significantly correlated with lymph node metastasis and advanced clinical stage. DAB2IP low expressing NPC patients showed a notable reduced overall survival and disease-free survival. Functionally, DAB2IP restoration prohibited cell proliferation, colony formation, migration and invasion in both 5-8F and CNE-2 cells. Moreover, DAB2IP overexpression restrained the subcutaneous growth and lung metastasis of NPC cells in nude mice. Mechanically, DAB2IP overexpression repressed the activation of phosphatidylinositol 3-kinase (PI3K)/Akt pathway and subsequently reduced the expression of its downstream targets including cyclin D1 and matrix metallopeptidase 7 (MMP7), which were identified as critical regulators for growth and metastasis of NPC. The Akt inhibitor MK-2206 showed similar effects to DAB2IP overexpression on growth and metastasis of 5-8F cells. Thus, DAB2IP suppresses growth and metastasis of NPC probably by targeting PI3K/Akt pathway, and may act as a hopeful therapeutic target for NPC.
\end{abstract}

\section{Introduction}

Nasopharyngeal carcinoma (NPC), which shows the highest morbidity in otorhinolaryngeal carcinoma, is one of the

Correspondence to: Dr Qianqian Gu, Department of Geriatrics, Cangzhou Central Hospital, 16 Xinhua West Road, Cangzhou, Hebei 061000, P.R. China

E-mail: czzxyy_gqq@163.com

Key words: DAB2IP, nasopharyngeal carcinoma, proliferation, metastasis, PI3K/Akt leading cause of cancer-related deaths all over the world (1). Although remarkable improvements have been achieved in the treatment of NPC, the prognosis for the NPC patients remains poor (2). Identifying novel molecules that are critical for the progression of NPC will bring new opportunity to find effective treatment options for NPC patients (3).

DAB2IP, a Ras GTPase-activating protein (4), exerts a critical role in cancer cell growth and metastasis as well as other aspects during tumor progression (5). Epigenetic silencing is the main cause of dysregulated expression of DAB2IP in human cancers (6). The expression of DAB2IP is silenced by promoter methylation and histone modification in prostate cancer $(7,8)$. In bladder cancer, DAB2IP expression is suppressed by posttranscriptionally regulation of miR-92b (9). Furthermore, DAB2IP is recognized as a direct downstream target of miR-889 in esophageal squamous cell carcinomas (10). In prostate cancer, loss of DAB2IP facilitates the metastasis and epithelial-mesenchymal transition (EMT) process of cancer cells (11), and DAB2IP silencing inhibits androgen deprivation therapy-induced apoptosis via targeting STAT3 signaling (12). Furthermore, DAB2IP deficiency promotes the growth of renal cell cancer (RCC) and leads to the resistance of mTOR-targeted therapies in metastatic RCC $(13,14)$. DAB2IP functions as a biomarker for prognosis prediction of colorectal cancer (CRC) and its loss promotes EMT and stem cell-like features in CRC cells $(15,16)$. However, the expression status and function of DAB2IP in NPC remain poorly known.

In this study, we found that DAB2IP was decreased in NPC tissues and cell lines. Decreased level of DAB2IP was correlated with malignant clinical features and poor prognosis of NPC patients. DAB2IP restoration inhibited the growth and metastasis of NPC cells in vitro and in vivo. Importantly, DAB2IP exerted its tumor suppressive effects on the growth and metastasis of NPC probably by targeting the PI3K/Akt pathway.

\section{Materials and methods}

Cell culture and transfection . NPC cell lines including CNE-1, CNE-2, 5-8F and 6-10B as well as a human immortalized nasopharyngeal epithelial cell line NP69 were from the Cell Bank of Chinese Academy of Sciences (Shanghai, China) and American Type Culture Collection (ATCC, Manassas, VA, 
USA). All cells were cultured in DMEM medium (Hyclone, Logan, UT, USA) supplemented with $10 \%$ fetal bovine serum (Hyclone). Cell cultures were kept in cell incubators with humidified atomosphere and $5 \% \mathrm{CO}_{2}$ at $37^{\circ} \mathrm{C}$. Retroviral vector pMMP-DAB2IP was constructed by inserting the corresponding cDNA into pMMP. The retroviruses were packaged and tranfected into CRC cells as previously described (17). Akt inhibitor MK-2206 (1 $\mu \mathrm{M}$, Selleck Chemicals, Houston, TX, USA) was used to treat NPC cells following the manufacturer's instructions.

Clinical samples. Clinical specimens including 68 NPC tissues and 20 non-cancerous nasopharyngeal epithelial tissues were collected at Cangzhou Central Hospital. Twenty non-cancerous nasopharyngeal epithelial tissues obtained at tonsillectomies were included as normal controls. Tissue specimens were conserved in liquid nitrogen. All these clinical tissues were pathologically confirmed before being used for further experiments in this study. NPC patients who previously received radiotherapy or chemotherapy were excluded. The informed consent was obtained from each patient involved in this study. Approvals for experiments involving the patient samples was obtained from the Institutional Research Ethics Committee of Cangzhou Central Hospital.

Quantitative real-time reverse transcription-polymerase chain reaction ( $q R T-P C R$ ). The total RNA from NPC tissues and cells were extracted with TRIzol (Invitrogen, Carlsbad, CA, USA). Reverse transcriptional reactions and PCR were performed with the Transcriptional First Strand cDNA Synthesis kit (Applied Biosystems, Foster City, CA, USA) and SYBR Green PCR Master Mix (Roche, Indianapolis, IN, USA). All primers including those for DAB2IP and GAPDH (internal control) were from Genecopoeia (Guangzhou, China). DAB2IP primers: 5'-TGG ACG ATG TGC TCT ATG CC-3' (forward), 5'-GGA TGG TGA TGG TTT GGT AG-3' (reverse); GAPDH primers: 5'-CAA GCT CAT TTC CTG GTA TGA C-3' (forward) and 5'-CAG TGA GGG TCT CTC TCT TCC T-3' (reverse). PCR amplification protocol was followed by $95^{\circ} \mathrm{C}(3 \mathrm{~min})$ and 36 cycles of amplification cycle $\left[95^{\circ} \mathrm{C}(30 \mathrm{sec}), 55^{\circ} \mathrm{C}(30 \mathrm{sec})\right.$, and $\left.72^{\circ} \mathrm{C}(1 \mathrm{~min})\right]$ using the ABI PRISM 7500 sequence detection system (Applied Biosystems). Data are presented as relative quantification based on the calculation of $2^{-\Delta \mathrm{Ct}} . \Delta \mathrm{Ct}$ was derived from subtracting the $\mathrm{Ct}$ value of reference cDNA from the $\mathrm{Ct}$ value of the cDNA of interest.

Western blot analysis. Total protein lysates $(30 \mu \mathrm{g})$ that were extracted from NPC tissues and cells with RIPA buffer were quantified with a BCA protein assay kit (Pierce, Bonn, Germany) and separated in 4-20\% SDS-PAGE gels. After being separated on gels, protein samples were transferred to PVDF membranes at $4^{\circ} \mathrm{C}$. The membranes were blocked with 5-10\% milk/TBST and were incubated with DAB2IP (1:500; sc-365921, Santa Cruz Biotechnology, Santa Cruz, CA, USA), p-AKT (1:2,000; \#4060, Ser473; Cell Signaling Technology, Beverly, MA, USA), AKT (1:1,000; \#4691, Cell Signaling Technology), cyclin D1 (1:1,000; \#2978, Cell Signaling Technology), MMP7 (1:800; 10374-2-AP, Proteintech, Rosemont, IL, USA) or GAPDH (1:1,000; sc-32233, Santa
Cruz Biotechnology) primary antibodies at $4^{\circ} \mathrm{C}$ overnight. Then, the membranes were incubated with secondary antibodies (1:5,000; \#7074/7076, Cell Signaling Technology). The protein signals on the membranes were detected using ECL reagents (Santa Cruz Biotechnology) and semi-quantified by ImageJ software (1.46; National Institutes of Health, Bethesda, MD, USA).

Proliferation assay. NPC cells that were treated with empty vector or DAB2IP were seeded into 96 -well plates $\left(1.5 \times 10^{3}\right.$ cells per well). CCK-8 cell viability assay were performed as previously reported (18). For the colony formation assay, 2,000 NPC cells were seeded on 6-well plates, and 14 to 21 days after cell seeding, cell colonies with crystal violet staining were counted.

Transwell assay. Transwell inserts were employed to evaluate the migratory and invasive ability of NPC cells. NPC cells $\left(1 \times 10^{4}\right)$ were resuspended in serum-free DMEM medium and they were seeded in the upper chamber. To induce the migration and invasion of NPC cells, the lower chambers were filled with $600 \mu \mathrm{l}$ DMEM supplemented with $20 \%$ FBS. Forty-eight hours after cell seeding, NPC cells migrated or invaded through the membranes (for invasion assay, the membrane were covered with $70 \mu 1$ Matrigel) were stained with crystal violet for cell counting under the microscope.

Subcutaneous implantation and tail vein injection assays. The in vivo growth ability of NPC cells was examined using the subcutaneous implantation nude mouse model using female $\mathrm{BALB} / \mathrm{c}$ nude mice (4-5 weeks). NPC cells $\left(1 \times 10^{6}\right)$ transfected with control vector or DAB2IP were subcutaneously injected into the left flank. During 24 days, the subcutaneous tumors were subjected to volume measurements and finally resected. To evaluate the in vivo metastatic ability of NPC cells, tail vein injection model was performed in the nude mice. NPC cells $\left(1 \times 10^{5}\right)$ transfected with control vector or DAB2IP were intravenous injected through the tail vein. Six weeks later, the mice were sacrificed by cervical dislocation under anesthesia. The lungs of nude mice were fixed with $10 \%$ formalin and paraffin-embedded, and were then subjected to $\mathrm{H} \& \mathrm{E}$ staining for potential lung metastatic lesions. All animal experiments in this study were approved by the Animal Care Committee of Cangzhou Central Hospital.

Statistical analysis. The data in this study are shown as mean \pm SEM. Statistical analysis including Student's t-test, Chi-square test, ANOVA, Kaplan-Meier analysis and log-rank test were performed using the GraphPad Prism 5 software (GraphPad Software, Inc, San Diego, CA, USA). P-value $<0.05$ was considered to be statistically significant.

\section{Results}

DAB2IP expression was decreased in NPC tissues and cells. To understand the expression status of DAB2IP in NPC, we extracted total proteins from the NPC tissues and noncancerous tissues. The results of immunoblotting showed that the levels of DAB2IP protein were significantly decreased in NPC tissues compared to non-cancerous nasopharyngeal 

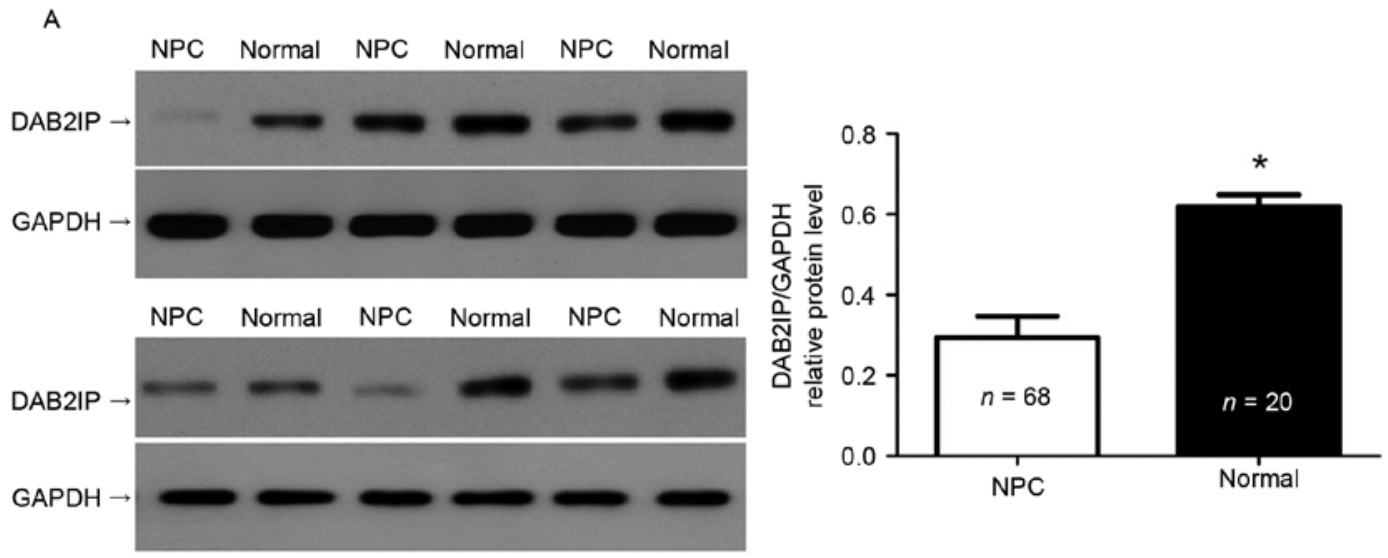

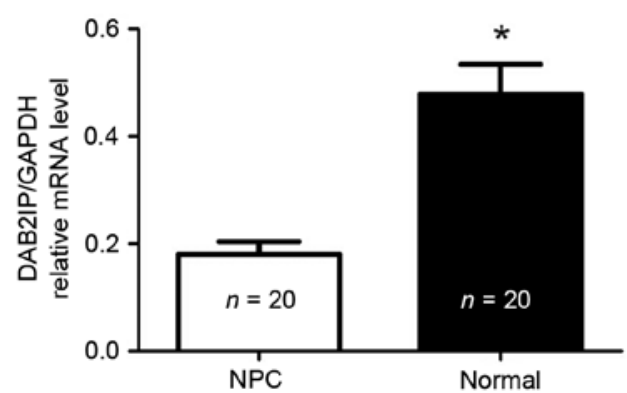

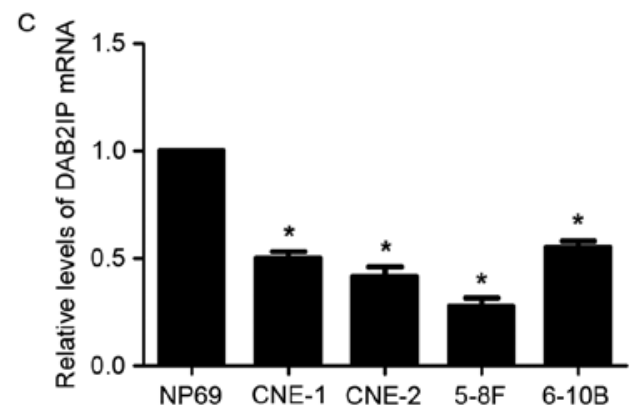

Figure 1. The status of DAB2IP expression in NPC. (A) The alterative expression of DAB2IP protein between NPC tissues ( $\mathrm{n}=68$ ) and normal nasopharyngeal epithelium tissues $(\mathrm{n}=20)$. " $\mathrm{P}<0.05$ by t-test. (B) The levels of DAB2IP mRNA in NPC tissues were significantly reduced compared to normal nasopharyngeal epithelium tissues. $n=20,{ }^{*} \mathrm{P}<0.05$ by t-test. (C) The differences in expression of four NPC cells lines (CNE-1, CNE-2, 5-8F and 6-10B) and a human immortalized nasopharyngeal epithelial cell line (NP69). $n=3$ replicates with similar results, ${ }^{*} \mathrm{P}<0.05$ by ANOVA.

epithelium tissues $(\mathrm{P}<0.05$, Fig. 1A). In addition, qRT-PCR was performed to detect the expression differences of DAB2IP mRNA in 20 paired NPC and normal tissues. DAB2IP mRNA was also notably underexpressed in NPC specimens $(\mathrm{P}<0.05$, Fig. 1B). Furthermore, we examined the expression of DAB2IP mRNA in NPC cell lines. Compared with NP69 cells, the expressions of DAB2IP mRNA was prominently lower in all NPC cell lines including CNE-1, CNE-2, 5-8F, and $6-10 \mathrm{~B}(\mathrm{P}<0.05$, respectively, Fig. 1C). Thus, downregulation of DAB2IP was confirmed in NPC.

Downregulation of DAB2IP correlates with unfavorable clinicopathological features and poor prognosis of NPC patients. After confirming the decreased expression of DAB2IP in NPC, we evaluated the clinical significance of DAB2IP in NPC patients. We divided the NPC patients into two groups (DAB2IP high or low group) based on the cutoff value which was defined as the median value of DAB2IP protein level. As shown in Table I, DAB2IP low expression was associated with advanced clinical stage $(\mathrm{P}=0.011)$ and lymph node metastasis $(\mathrm{P}=0.006)$. Furthermore, DAB2IP low expressing NPC patients showed a notable reduced 5-year overall survival and disease-free survival $(\mathrm{P}=0.045$ and $\mathrm{P}=0.032$, respectively, Fig. 2). These data indicate that DAB2IP is a promising prognostic marker for NPC patients.

$D A B 2 I P$ restrains the proliferation, migration and invasion of NPC cells. Since the levels of DAB2IP mRNA were relatively lower in 5-8F and CNE-2 cells, DAB2IP expression was
Table I. Correlation between the clinicopathological features and DAB2IP expression in nasopharyngeal carcinoma.

\begin{tabular}{lcccc}
\hline & & \multicolumn{2}{c}{ DAB2IP expression } & \\
Characteristics & $\mathrm{n}$ & Low (n=34) & High (n=34) & P-value \\
\hline Age (years) & & & & 0.625 \\
$<50$ & 38 & 20 & 18 & \\
$\geq 50$ & 30 & 14 & 16 & \\
Sex & & & & 0.442 \\
$\quad$ Male & 45 & 21 & 24 & \\
Female & 23 & 13 & 10 & \\
Clinical stage & & & & $0.011^{\mathrm{a}}$ \\
I+II & 12 & 2 & 10 & \\
III+IV & 56 & 32 & 24 & \\
Lymph node & & & & $0.006^{\mathrm{a}}$ \\
metastasis & & & & \\
No & 18 & 4 & 14 & \\
Yes & 50 & 30 & 20 & \\
Distant & & & & 0.132 \\
metastasis & & & & \\
No & 60 & 28 & 32 & \\
Yes & 8 & 6 & 2 & \\
\hline
\end{tabular}

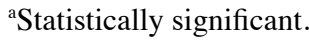




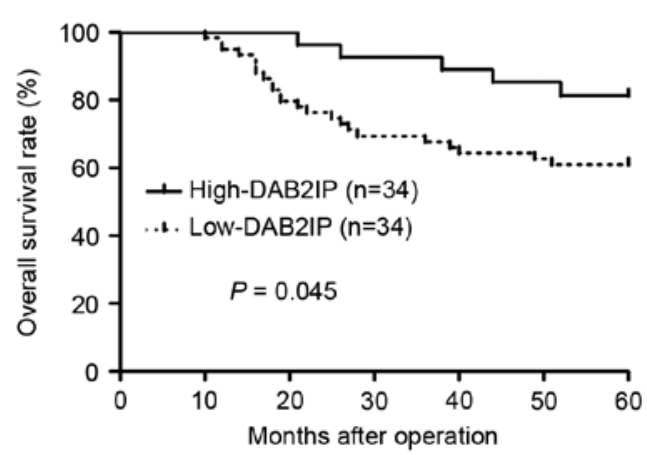

B

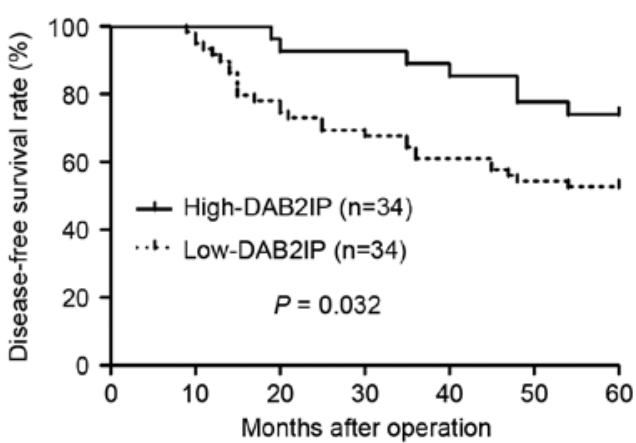

Figure 2. The prognostic value of DAB2IP in NPC patients. The NPC patients were divided into two groups (DAB2IP high or low group, $\mathrm{n}=34$ respectively) based on the cutoff value which was defined as the median value of DAB2IP protein level. Compared with those of high DAB2IP level, DAB2IP low-expressing NPC patients had a notable reduced 5 -year (A) overall survival and (B) disease-free survival rate. $\mathrm{P}<0.05$ by log-rank test.

A

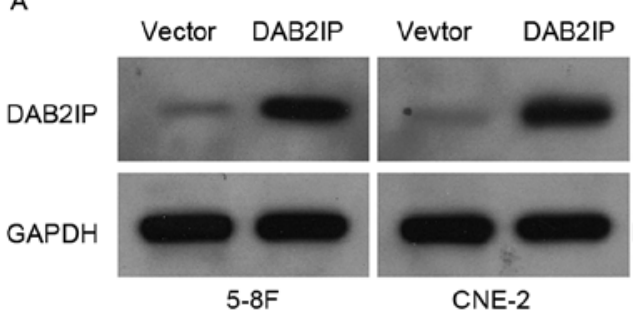

C

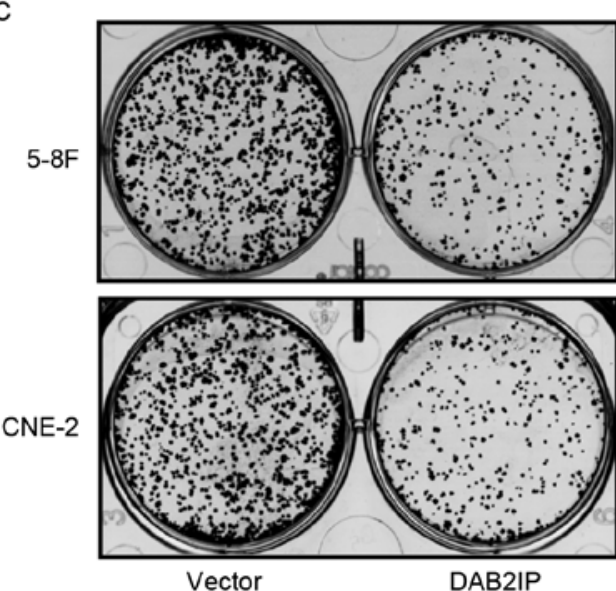

B
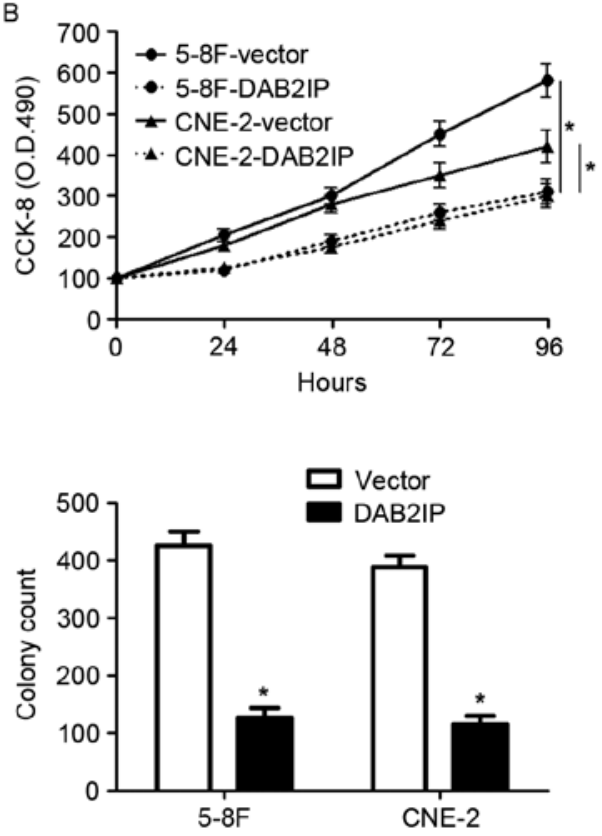

Figure 3. DAB2IP restoration restrains the proliferation of NPC cells. (A) 5-8F and CNE-2 cells that were infected with control vector or DAB2IP retroviruses were confirmed by immunoblotting. (B) CCK-8 assays confirmed that DAB2IP restoration obviously prohibited proliferation of both 5-8F and CNE-2 cells $\mathrm{n}=3$ replicates with similar results, ${ }^{*} \mathrm{P}<0.05$ by ANOVA. (C) The relative colony numbers were evidently reduced after DAB2IP restoration in both $5-8 \mathrm{~F}$ and CNE- 2 cells. $n=3$ replicates with similar results, ${ }^{*} \mathrm{P}<0.05$ by t-test.

restored by retroviruses infection in these two cells (Fig. 3A). CCK-8 assays revealed that overexpression of DAB2IP significantly inhibited the proliferation of both $5-8 \mathrm{~F}$ and $\mathrm{CNE}-2$ cells $(\mathrm{P}<0.05$, respectively, Fig. 3B). In addition, the number of colonies was notably reduced after DAB2IP overexpression in NPC cells $(\mathrm{P}<0.05$, respectively, Fig. 3C). Transwell assays showed that DAB2IP overexpression obviously decreased the number of migrated and invaded cells $(\mathrm{P}<0.05$, respectively, Fig. 4). Thus, DAB2IP suppresses NPC cell proliferation, migration and invasion in vitro.

$D A B 2 I P$ reduces growth and metastasis of NPC cells in nude mice. To further confirm the functional influence of DAB2IP on NPC cells, we performed subcutaneous tumor formation and lung metastasis experiments to test whether DAB2IP affected growth and metastasis of NPC cells. Overexpression of DAB2IP obviously decreased the subcutaneous growth of NPC in nude mice as suggested by tumor growth curves $(\mathrm{P}<0.05$, Fig. 5A). In addition, lung metastasis experiments showed that DAB2IP notably reduced the number of metastatic nodules in the lungs of nude mice $(\mathrm{P}<0.05$, Fig. 5B). Altogether, DAB2IP functions as a tumor suppressor in NPC.

PI3K/Akt pathway is a potential downstream target of $D A B 2 I P$ in NPC cells. After determining the biological functions of DAB2IP in NPC cells, we examined the underlying 
A
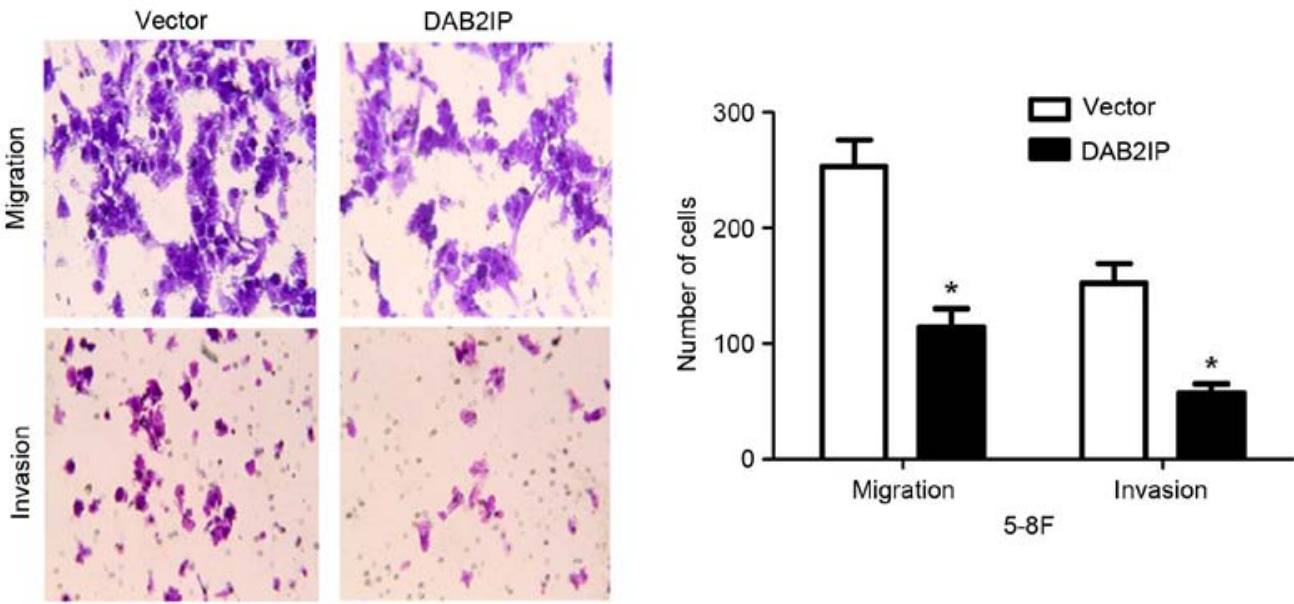

B
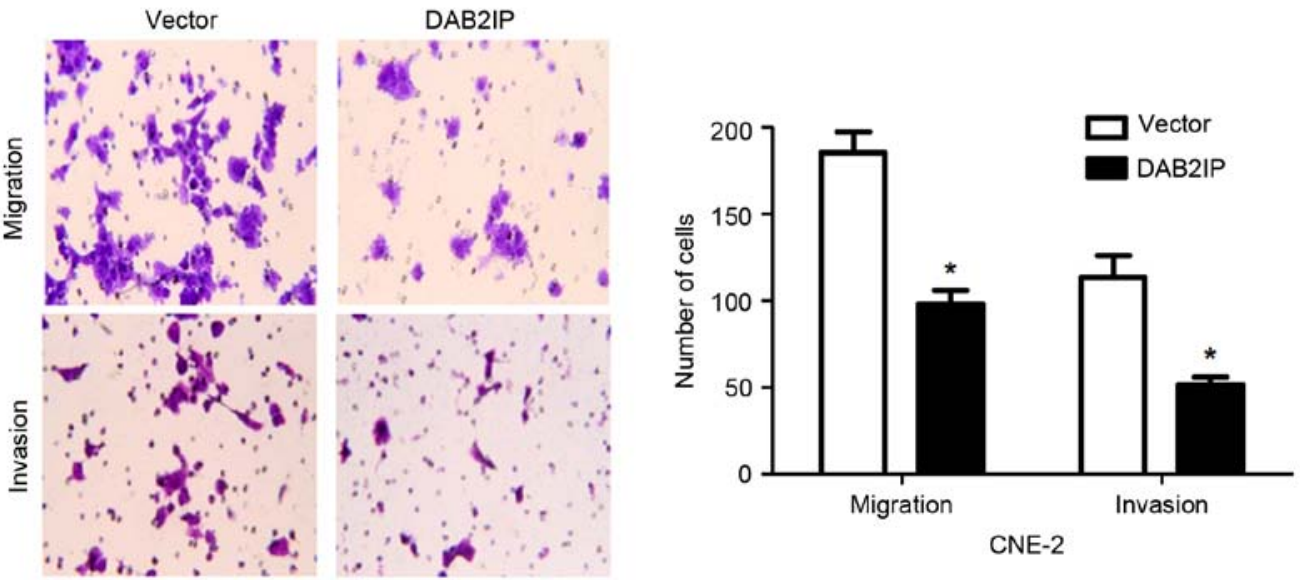

Figure 4. DAB2IP overexpression inhibits the migration and invasion of NPC cells. (A) Transwell assays confirmed that DAB2IP overexpression significantly reduced the migration and invasion of $5-8 \mathrm{~F}$ cells. $\mathrm{n}=3$ replicates with similar results, "P<0.05 by $\mathrm{t}$-test. (B) The number of migrated and invaded cells was prominently reduced after DAB2IP overexpression in CNE- 2 cells. $n=3$ replicates with similar results, ${ }^{*} \mathrm{P}<0.05$ by $\mathrm{t}$-test.

A
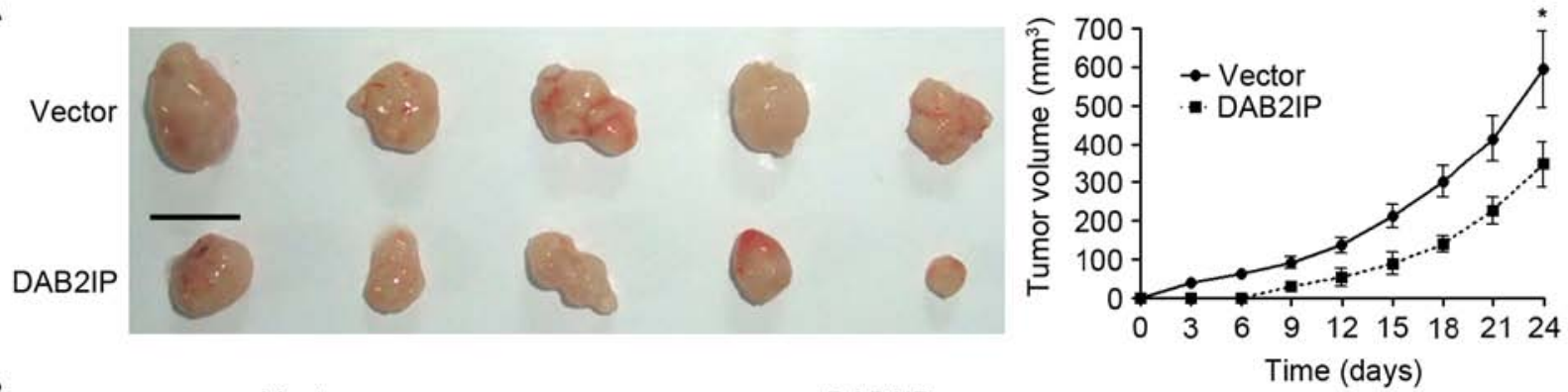

B

Vector

DAB2IP

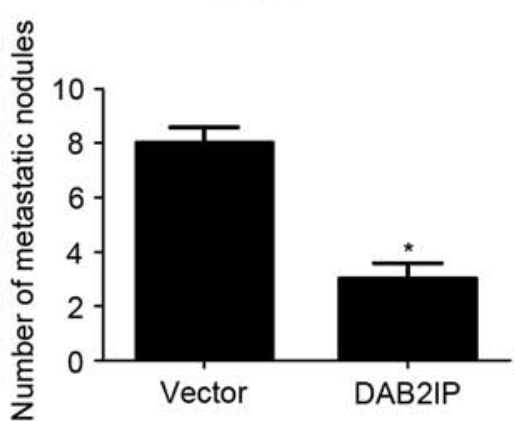

Figure 5. DAB2IP inhibits growth and lung metastasis of NPC in nude mice. (A) $5-8 \mathrm{~F}$ cells that were infected with control vector or DAB2IP retroviruses were implanted into nude mice via subcutaneous injection. Tumor growth curves revealed that DAB2IP overexpression significantly inhibited the growth of NPC in vivo. $\mathrm{n}=5,{ }^{*} \mathrm{P}<0.05$ by ANOVA. Scale bar, $1 \mathrm{~cm}$. (B) $5-8 \mathrm{~F}$ cells that were infected with control vector or DAB2IP retroviruses were intravenously injected into nude mice via tail vein. $\mathrm{H} \& \mathrm{E}$ staining revealed that DAB2IP overexpression significantly reduced lung metastases of 5-8F cells (original magnification, $\mathrm{x} 100) . \mathrm{n}=5,{ }^{*} \mathrm{P}<0.05$ by t-test. $\mathrm{M}$, metastases; $\mathrm{N}$, normal lung. 


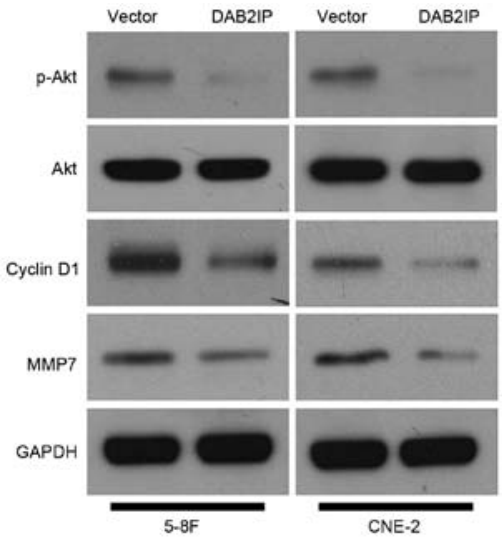

Figure 6. DAB2IP regulates the activation of PI3K/Akt pathway in NPC cells. The activity of PI3K/Akt pathway and the levels of its downstream proteins, cyclin D1 and MMP7, after DAB2IP overexpression were examined by western blot analysis in both 5-8F and CNE-2 cells. DAB2IP overexpression prohibited the activation of PI3K/Akt pathway with reduced expression of phosphorylated Akt and downregulated the levels of cyclin D1 and MMP7 in NPC cells. mechanisms involved in tumor suppressive role of DAB2IP. DAB2IP has been reported to regulate various signaling pathways including PI3K/Akt pathway in human cancer (5). Furthermore, the downstream targets of PI3K/Akt pathway such as cyclin D1 and MMP7 regulate proliferation, migration and invasion in NPC cells $(19,20)$. Subsequently, 5-8F and CNE-2 cells that were infected with empty vector or DAB2IP retroviruses were subjected to immunoblotting for $\mathrm{p}-\mathrm{Akt}$, Akt, cyclin D1 and MMP7. Our results revealed that DAB2IP repressed the activation of PI3K/Akt pathway with reduced level of phosphorylated Akt in NPC cells (Fig. 6). Importantly, the expressions of cyclin D1 and MMP7 was accordingly downregulated in both 5-8F and CNE-2 cells (Fig. 6). Next, 5-8F cells that were treated with MK-2206, a known Akt inhibitor, were subjected to growth and metastasis assays. Western blot analysis indicated that MK-2206 obviously reduced the levels of phosphorylated Akt and its downstream proteins, cyclin D1 and MMP7 (Fig. 7A). In addition, MK-2206 significantly reduced the proliferation, migration and invasion of
A

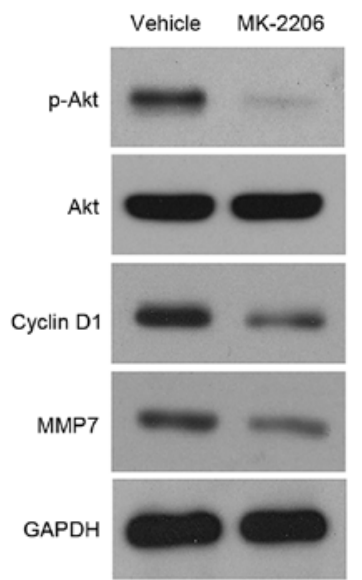

C

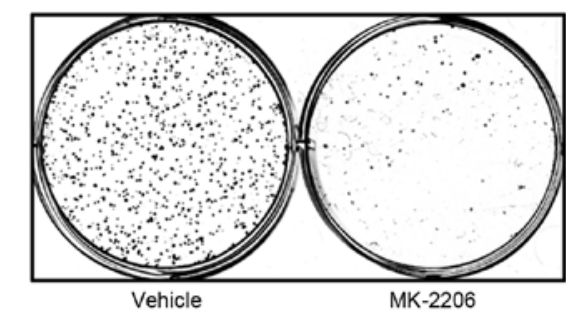

D

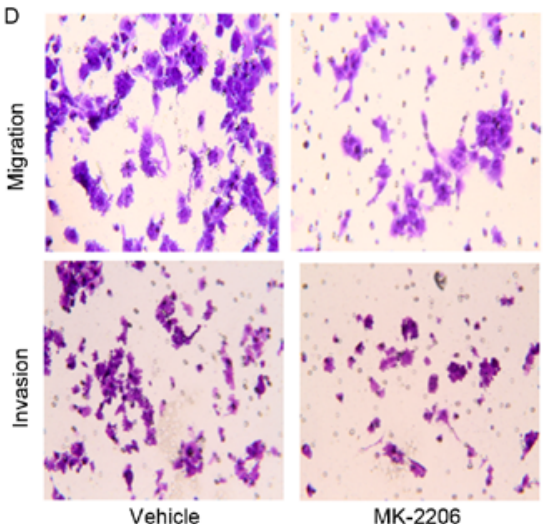

B
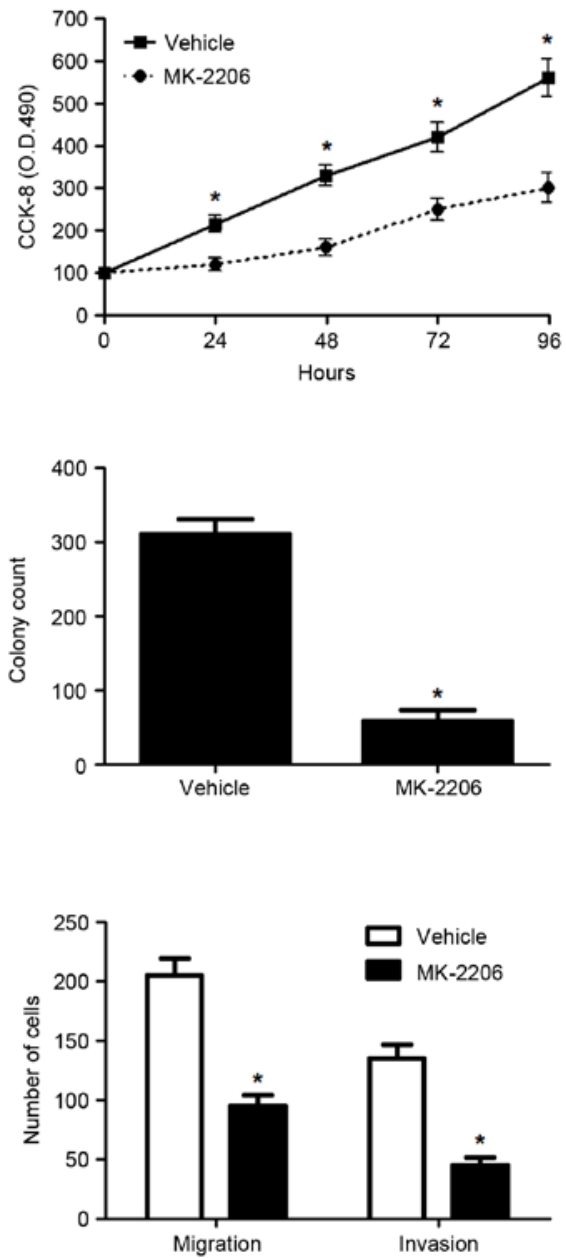

Figure 7 Akt inhibitor MK-2206 inhibits the proliferation and metastasis of 5-8F cells. (A) 5-8F cells that were treated with vehicle or MK-2206 (1 $\mu$ M) were confirmed by western blotting for p-Akt, Akt, cyclin D1 and MMP7 expression. (B) CCK-8 assays confirmed that MK-2206 treatment obviously inhibited proliferation of $5-8 \mathrm{~F}$ cells. $\mathrm{n}=3$ replicates with similar results, ${ }^{*} \mathrm{P}<0.05$ by ANOVA. (C) The relative colony numbers were notably reduced after MK-2206 treatment in $5-8 \mathrm{~F}$ cells. $\mathrm{n}=3$ replicates with similar results, ${ }^{*} \mathrm{P}<0.05$ by t-test. (D) Transwell assays indicated that MK-2206 treatment prominently restrained the migration and invasion of $5-8 \mathrm{~F}$ cells. $\mathrm{n}=3$ replicates with similar results, ${ }^{*} \mathrm{P}<0.05$ by $\mathrm{t}$-test. 
$5-8 \mathrm{~F}$ cells $(\mathrm{P}<0.05$, respectively, Fig. 7B-D). Here, we suggest that DAB2IP suppresses the progression of NPC possibly by targeting the PI3K/Akt pathway.

\section{Discussion}

Loss or downregulation of tumor suppressors have been found to be closely associated with cancer development and progression $(21,22)$. Among these tumor suppressors, DAB2IP is a newly identified tumor suppressor closely related with human cancers (5). In this study, we confirmed for the first time that DAB2IP expression was significantly reduced in NPC tissues and cell lines. Decreased DAB2IP level was associated with unfavorable clinicopathological features and poor prognosis of NPC patients. In vitro functional assays showed that DAB2IP could restrain proliferation, migration and invasion of NPC cells. In vivo experiments also confirmed that DAB2IP prohibited the growth and metastasis of NPC cells in nude mice. These data indicate that DAB2IP plays a tumor suppressive role in NPC by inhibiting tumor growth and metastasis.

DAB2IP was identified as a new member of the Ras GTPase-activation protein family (5). DAB2IP regulates cell proliferation, apoptosis, migration, invasion and survival via modulating various signaling pathways by directly interacting with DAB2 (5). Previous studies showed DAB2IP exerts critical roles in human cancers including prostate cancer $(6,8,12)$, CRC $(15,16)$ and RCC $(13,14)$. PI3K/Akt pathway has been reported to play an important role in the initiation and progression of NPC $(23,24)$. Notably, DAB2IP suppression of the activation of PI3K/Akt pathway is reported in prostate cancer (25), bladder cancer (26), but not in NPC. Here, we revealed that DAB2IP overexpression blocked that activation of PI3K/Akt pathway with reduced level of phosphorylated Akt in NPC cells. Accordingly, the levels of cyclin D1 (27) and MMP7 (28), downstream targets of PI3K/Akt pathway, were downregulated by DAB2IP restoration. cyclin D1, a regulator of cell cycle progression, promotes NPC cell proliferation via promoting G (1)-phase transition (29). Moreover, MMP7 has been reported to promote invasion of NPC cells (20). A previous study suggested that Wnt signaling facilitates cell proliferation via cyclin D1 and promoted invasion by MMP7 in NPC (20). The expression of PI3K/Akt pathway molecules, such as p-Akt, cyclin D1 and MMP7, were upregulated in NPC tissues compared to normal tissues as previously reported (30-32). Furthermore, MK-2206, the Akt inhibitor (33), blocked the PI3K/Akt pathway and suppressed the growth and metastasis of NPC cells in vitro, which was consistent with a previous study (34). Thus, we suggest that DAB2IP restrains growth and metastasis of NPC by inhibiting PI3K/Akt pathway and subsequently suppressing cyclin D1 and MMP7 expression. DAB2IP expression is frequently impaired by methylation in cancer (35). Thus, the methylation status of DA2BIP in NPC is worth investigation in the future.

Collectively, DAB2IP expression was decreased in NPC tissues and cells. DAB2IP inhibited the progression of NPC by suppressing the proliferation and metastasis of NPC cells both in vitro and in vivo. Mechanically, PI3K/Akt was identified to be the downstream target of DAB2IP in NPC cells. DAB2IP potentially acts as a prognostic predictor and a drug-target for NPC patients.

\section{Acknowledgements}

The authors thank all the patients who participated in this study.

\section{References}

1. Ou SH, Zell JA, Ziogas A and Anton-Culver H: Epidemiology of nasopharyngeal carcinoma in the United States: Improved survival of Chinese patients within the keratinizing squamous cell carcinoma histology. Ann Oncol 18: 29-35, 2007.

2. Lee AW, Ma BB, Ng WT and Chan AT: Management of nasopharyngeal carcinoma: Current practice and future perspective. J Clin Oncol 33: 3356-3364, 2015.

3. Bruce JP, Yip K, Bratman SV, Ito E and Liu FF: Nasopharyngeal cancer: Molecular landscape. J Clin Oncol 33: 3346-3355, 2015.

4. Wang Z, Tseng CP, Pong RC, Chen H, McConnell JD, Navone N and Hsieh JT: The mechanism of growth-inhibitory effect of DOC-2/DAB2 in prostate cancer. Characterization of a novel GTPase-activating protein associated with N-terminal domain of DOC-2/DAB2. J Biol Chem 277: 12622-12631, 2002.

5. Liu L, Xu C, Hsieh JT, Gong J and Xie D: DAB2IP in cancer. Oncotarget 7: 3766-3776, 2016.

6. Tsai YS, Lai CL, Lai CH, Chang KH, Wu K, Tseng SF, Fazli L, Gleave M, Xiao G, Gandee L, et al: The role of homeostatic regulation between tumor suppressor DAB2IP and oncogenic Skp2 in prostate cancer growth. Oncotarget 5: 6425-6436, 2014.

7. Chen H, Toyooka S, Gazdar AF and Hsieh JT: Epigenetic regulation of a novel tumor suppressor gene (hDAB2IP) in prostate cancer cell lines. J Biol Chem 278: 3121-3130, 2003.

8. Chen H, Tu SW and Hsieh JT: Down-regulation of human DAB2IP gene expression mediated by polycomb Ezh2 complex and histone deacetylase in prostate cancer. J Biol Chem 280: 22437-22444, 2005.

9. Huang J, Wang B, Hui K, Zeng J, Fan J, Wang X, Hsieh JT, He D and $\mathrm{Wu} \mathrm{K}$ : miR-92b targets DAB2IP to promote EMT in bladder cancer migration and invasion. Oncol Rep 36: 1693-1701, 2016.

10. Xu Y, He J, Wang Y, Zhu X, Pan Q, Xie Q and Sun F: miR-889 promotes proliferation of esophageal squamous cell carcinomas through DAB2IP. FEBS Lett 589: 1127-1135, 2015.

11. Wang B, Huang J, Zhou J, Hui K, Xu S, Fan J, Li L, Wang X, Hsieh JT, He D, et al: DAB2IP regulates EMT and metastasis of prostate cancer through targeting PROX1 transcription and destabilizing HIF1 $\alpha$ protein. Cell Signal 28: 1623-1630, 2016.

12. Zhou J, Ning Z, Wang B, Yun EJ, Zhang T, Pong RC, Fazli L, Gleave M, Zeng J, Fan J, et al: DAB2IP loss confers the resistance of prostate cancer to androgen deprivation therapy through activating STAT3 and inhibiting apoptosis. Cell Death Dis 6: e1955, 2015.

13. Zhou J, Luo J, Wu K, Yun EJ, Kapur P, Pong RC, Du Y, Wang B, Authement C, Hernandez E, et al: Loss of DAB2IP in RCC cells enhances their growth and resistance to mTOR-targeted therapies. Oncogene 35: 4663-4674, 2016.

14. Yeh CR, Ou ZY, Xiao GQ, Guancial E and Yeh S: Infiltrating $\mathrm{T}$ cells promote renal cell carcinoma ( $\mathrm{RCC}$ ) progression via altering the estrogen receptor $\beta$-DAB2IP signals. Oncotarget 6: 44346-44359, 2015.

15. Min J, Liu L, Li X, Jiang J, Wang J, Zhang B, Cao D, Yu D, Tao D, Hu J, et al: Absence of DAB2IP promotes cancer stem cell like signatures and indicates poor survival outcome in colorectal cancer. Sci Rep 5: 16578, 2015.

16. Wang J, Zhu X, Hu J, He G, Li X, Wu P, Ren X, Wang F, Liao W, Liang L, et al: The positive feedback between Snail and DAB2IP regulates EMT, invasion and metastasis in colorectal cancer. Oncotarget 6: 27427-27439, 2015.

17. Tu K, Yang W, Li C, Zheng X, Lu Z, Guo C, Yao Y and Liu Q: Fbxw7 is an independent prognostic marker and induces apoptosis and growth arrest by regulating YAP abundance in hepatocellular carcinoma. Mol Cancer 13: 110, 2014.

18. Shao J, Yang X, Liu T, Zhang T, Xie QR and Xia W: Autophagy induction by SIRT6 is involved in oxidative stress-induced neuronal damage. Protein Cell 7: 281-290, 2016.

19. Wu M, Ye X, Deng X, Wu Y, Li X and Zhang L: Upregulation of metastasis-associated gene 2 promotes cell proliferation and invasion in nasopharyngeal carcinoma. Onco Targets Ther 9: $1647-1656,2016$ 
20. Wong AM, Kong KL, Chen L, Liu M, Wong AM, Zhu C, Tsang JW and Guan XY: Characterization of CACNA2D3 as a putative tumor suppressor gene in the development and progression of nasopharyngeal carcinoma. Int J Cancer 133: 2284-2295, 2013.

21. Hazan I, Hofmann TG and Aqeilan RI: Tumor suppressor genes within common fragile sites are active players in the DNA damage response. PLoS Genet 12: e1006436, 2016.

22. Paysan L, Piquet L, Saltel F and Moreau V: Rnd3 in cancer: A review of the evidence for tumor promoter or suppressor. Mol Cancer Res 14: 1033-1044, 2016.

23. Zhao M, Luo R, Liu Y, Gao L, Fu Z, Fu Q, Luo X, Chen Y, Deng X, Liang Z, et al: miR-3188 regulates nasopharyngeal carcinoma proliferation and chemosensitivity through a FOXO1-modulated positive feedback loop with mTOR-p-PI3K/AKT-c-JUN. Nat Commun 7: 11309, 2016.

24. Yang CF, Yang GD, Huang TJ, Li R, Chu QQ, Xu L, Wang MS, Cai MD, Zhong L, Wei HJ, et al: EB-virus latent membrane protein 1 potentiates the stemness of nasopharyngeal carcinoma via preferential activation of PI3K/AKT pathway by a positive feedback loop. Oncogene 35: 3419-3431, 2016.

25. Xie D, Gore C, Zhou J, Pong RC, Zhang H, Yu L, Vessella RL, Min W and Hsieh JT: DAB2IP coordinates both PI3K-Akt and ASK1 pathways for cell survival and apoptosis. Proc Natl Acad Sci USA 106: 19878-19883, 2009.

26. Shen YJ, Kong ZL, Wan FN, Wang HK, Bian XJ, Gan HL, Wang CF and Ye DW: Downregulation of DAB2IP results in cell proliferation and invasion and contributes to unfavorable outcomes in bladder cancer. Cancer Sci 105: 704-712, 2014.

27. Zhuang YJ, Liao ZW, Yu HW, Song XL, Liu Y, Shi XY, Lin XD and Zhou TC: ShRNA-mediated silencing of the ubiquitinspecific protease 22 gene restrained cell progression and affected the Akt pathway in nasopharyngeal carcinoma. Cancer Biol Ther 16: 88-96, 2015.
28. Xu Z, Liu D, Fan C, Luan L, Zhang X and Wang E: DIXDC1 increases the invasion and migration ability of non-small-cell lung cancer cells via the PI3K-AKT/AP-1 pathway. Mol Carcinog 53: 917-925, 2014.

29. Zhang LY, Ho-Fun Lee V, Wong AM, Kwong DL, Zhu YH, Dong SS, Kong KL, Chen J, Tsao SW, Guan XY, et al: MicroRNA-144 promotes cell proliferation, migration and invasion in nasopharyngeal carcinoma through repression of PTEN. Carcinogenesis 34: 454-463, 2013.

30. Yip WK, Leong VC, Abdullah MA, Yusoff S and Seow HF. Overexpression of phospho-Akt correlates with phosphorylation of EGF receptor, FKHR and BAD in nasopharyngeal carcinoma. Oncol Rep 19: 319-328, 2008.

31. Lai JP, Tong CL, Hong C, Xiao JY, Tao ZD, Zhang Z, Tong WM and Betz CS: Association between high initial tissue levels of cyclin $\mathrm{d} 1$ and recurrence of nasopharyngeal carcinoma. Laryngoscope 112: 402-408, 2002.

32. Chen J, Kwong DL, Zhu CL, Chen LL, Dong SS, Zhang LY, Tian J, Qi CB, Cao TT, Wong AM, et al: RBMS3 at 3p24 inhibits nasopharyngeal carcinoma development via inhibiting cell proliferation, angiogenesis, and inducing apoptosis. PLoS One 7: e44636, 2012.

33. Ma BB, Lui VW, Hui CW, Lau CP, Wong CH, Hui EP, Ng MH, Tsao SW, Li Y and Chan AT: Preclinical evaluation of the AKT inhibitor MK-2206 in nasopharyngeal carcinoma cell lines. Invest New Drugs 31: 567-575, 2013.

34. Hu W, Xiao L, Cao C, Hua S and Wu D: UBE2T promotes nasopharyngeal carcinoma cell proliferation, invasion, and metastasis by activating the AKT/GSK $3 \beta / \beta$-catenin pathway. Oncotarget 7 : 15161-15172, 2016.

35. Bellazzo A, Di Minin G and Collavin L: Block one, unleash a hundred. Mechanisms of DAB2IP inactivation in cancer. Cell Death Differ 24: 15-25, 2017. 